conditions of loading, it was found that vertical loads placed on a group of shot (ball or spheres of equal diameter) are very effective as a practical method of flattening the resonance curve; the load on the shot acts as a very good damper of the vibration, and is more effective the nearer to the roof it is placed.

Fig. 1 shows the free vibration curve of a frame structure $(4 \times 20 \mathrm{~cm} .=80$ $\mathrm{cm}$. high, $20 \mathrm{~cm}$. bay and $1 \mathrm{~cm}$. thick), the load conditions and period of which are indicated in the figure. Fig. 2 shows the same free vibration



Fig. 1.

Direct LOAD, $435 \mathrm{gm}$; DEAd LOAD, $860 \mathrm{gm}$.; PERIod, 0.125 sec. sheet bent to the shape of a longitudinal section of the actual electrode.

A trough of the type described gives very satis. factory results if a few minor precautions are taken. It is necessary to produce a contact angle between the liquid and insulating bottom which approximates to the angle of the wedge as nearly as possible. This is obtained by allowing the liquid to wet the bottom of the trough only up to the axis. Then by reducing the angle of tilt the desired contact angle can be got. The scale of the system is made as large as possible so that it is unnecessary to plot the field in the neighbourhood of the axis where capillary rise on the probe would introduce serious error. In addition to the above-mentioned precautions it is, of course, necessary to observe all the usual points in working with an A.c. electrolytic bridge.

M. Bowman-Manifold.

F. H. NrcoLr.

Research Laboratories,

Electric and Musical Industries, Ltd., Hayes, Middlesex. May 27.



curve damped by an indirect load on ball-bearings, the load conditions of which are indicated in the figure. These damping characteristics are the principal object of the studies described in the present note.

I wish to express my thanks to the Council of the Japan Society for the Promotion of Scientific Research, with the aid of which the present

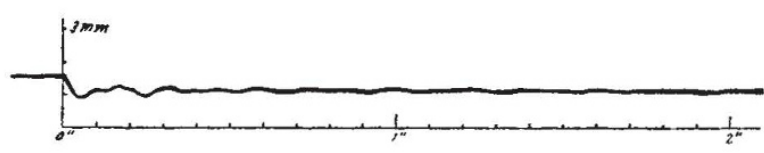

Fig. 2.

INDIRECT LOAD ON BALL-BEARINGS, 400 gm.; WEIGHT OF BALLBEARINGS, $35 \mathrm{gm}$.; DEAD LOAD, $860 \mathrm{gm}$.

investigation, including some hundred model experiments of a similar kind, has been made possible.

Hokkaido Imperial University, F. TAKabeya. Sapporo.

\section{Electrolytic Field Plotting Trough for Circularly Symmetric Systems}

THE method of plotting electrostatic equipotentials between conductors by measuring the potentials in a geometrically similar problem in conduction is very old and well known. We have applied this to a modified form of electrolytic trough which we have been using in this laboratory for the past four years. This has been specially designed for circularly symmetric systems.

It is always permissible in problems of conduction to introduce along any surface an insulating barrier across which there is no flow of current. We have applied this to circularly symmetric systems by cutting the system along two diametrical planes, thus obtaining a liquid wedge. Such a wedge is realized in practice by a tilted trough containing electrolyte resting on a plane insulating bottom. If the angle of the wedge is small, the electrodes used in it need not be surfaces of revolution but can be replaced by pieces of metal

\section{Free Field Calibration of Microphones}

Is connexion with the contribution by Messrs. King and Maguire to this subject in Nature of June 4, p. 1016, it may be of interest to refer to a very similar method of calibration which has been in use by the Research Branch of the Post Office Engineering Department since 1933.

The 'probe tube' device to which I refer ${ }^{1}$ is so constructed that reflections of sound in the tube are practically eliminated. This means that the acoustical impedance presented by the open end of the tube is substantially non-reactive. The open end can therefore be exposed at one of the closed ends of the resonant air column which is used for calibrating, without affecting the positions of the nodes and anti-nodes in the stationary wave system set up in the air column. The air particle velocity is measured by a Rayleigh disk located at the centre of the air column at any frequency of resonance at which the length of the column is an odd multiple of half a wave-length, and the sound pressure at the end of the column, that is, at the open end of the probe tube, is thus known. Since the tube is small, the calibration is the same as a free field calibration.

With an air column about seven feet in length, calibration can be effected at more than forty values of frequency, extending from 80 to more than 6,400 cycles per second, without any readjustment of either the length of the air column or the position of the probe tube.

The absence of resonance in the probe tube enables the device to be used for measuring sound pressures at a point, not only in an unobstructed sound field, but also at the surfaces of solid obstacles or near the openings of resonators, without appreciably altering the pattern of the sound field.

Post Office Research Station, W. West. Dollis Hill,

London, N.W.2. June 13.

${ }^{1}$ West, W., Post Office Elec. Eng. J., 28, 260 (Jan. 1934). 